\title{
Constraining the thermal history of the warm-hot intergalactic medium
}

\author{
L. Zappacosta ${ }^{1}$, R. Maiolino ${ }^{2}$, A. Finoguenov ${ }^{3}$, F. Mannucci ${ }^{4}$, R. Gilli ${ }^{2}$, and A. Ferrara ${ }^{5}$ \\ 1 Dipartimento di Astronomia e Scienza dello Spazio, Largo E. Fermi 2, 50125 Firenze, Italy \\ e-mail: zappacos@nabhas.ps.uci.edu \\ 2 Osservatorio Astrofisico di Arcetri Largo E. Fermi 5, 50125 Firenze, Italy \\ 3 Max-Planck-Institut für extraterrestrische Physik, Giessenbachstraße, 85748 Garching, Germany \\ ${ }^{4}$ Istituto di Radioastronomia, Sezione di Firenze - CNR Largo E. Fermi 5, 50125 Firenze, Italy \\ 5 SISSA/International School for Advanced Studies via Beirut, 434014 Trieste, Italy
}

Received 2 March 2004 / Accepted 22 December 2004

\begin{abstract}
We have identified a large-scale structure traced by galaxies at $z=0.8$, within the Lockman Hole, by means of multi-object spectroscopic observations. By using deep XMM images we have investigated the soft X-ray emission from the Warm-Hot Intergalactic Medium (WHIM) expected to be associated with this large-scale structure and we set a tight upper limit to its flux in the very soft $0.2-0.4 \mathrm{keV}$ band. The non-detection requires the WHIM at these redshifts to be cooler than $0.1 \mathrm{keV}$. Combined with the WHIM emission detections at lower redshift, our result indicates that the WHIM temperature rapidly decreases with redshift, as expected in popular cosmological models.
\end{abstract}

Key words. cosmology: large-scale structure of Universe - X-rays: diffuse background - galaxies: intergalactic medium galaxies: distances and redshifts

\section{Introduction}

Both observations and cosmological models suggest that most of the baryonic matter is located in the intergalactic medium (IGM). Cosmological models have also shown that the evolution of the baryonic matter is driven by progressive gravitational heating in the potential field of dark matter filaments (Cen \& Ostriker 1999; Davé et al. 2001). In particular, at high redshifts $(z>2-3)$ the baryonic gas is relatively cold $\left(T<10^{5} \mathrm{~K}\right)$, and is identified with Ly $\alpha$ absorbers along the line of sight of quasars, while at lower redshifts $(z<1)$ the shocks due to the infall of the gas on the dark matter filaments (traced by the regions of high galaxy number densities) gradually heat the gas to temperatures in the range $T \sim 10^{5}-10^{7} \mathrm{~K}$.

The identification of such Warm-Hot Intergalactic Medium (WHIM) in the local universe has received growing interest in the last few years. The detection of absorption lines from highly ionized species (O VI, O VII, O VIII), both in the UV and in the soft X-rays, has allowed investigators to unambiguosly identify WHIM along the line of sight of a few bright quasars (Nicastro et al. 2002; Mathur et al. 2003). The detection of emission due to WHIM is quite challenging. Indeed, the WHIM is expected to emit weak and diffuse radiation mostly in the softest X-ray bands $(\$ 1 \mathrm{keV})$, where both the Galactic absorption and the foreground emission from the Local Hot Bubble (LHB) and Milky Way Halo are strong. Nevertheless, several independent detections of WHIM emission were obtained by detailed analysis of soft X-ray maps (ROSAT and XMM) in regions characterized by galaxy overdensities and by the spectral analysis of clusters of galaxies and of their surroundings (Wang et al. 1997; Sołtan et al. 2002; Zappacosta et al. 2002, 2005; Kaastra et al. 2003; Finoguenov et al. 2003).

All WHIM detections discussed above have been obtained for gas in the local universe or at low redshift. The most distant WHIM emission detected so far was obtained at $z \sim 0.45$ by Zappacosta et al. (2002). At higher redshift the detection is more difficult for both technical and physical reasons. Indeed, the lack of bright, high redshift quasars prevents the detection of WHIM features in absorption, while the thermal cutoff is redshifted to lower energies making it more difficult to detect the WHIM emission even in the soft X-rays. An additional issue is that, according to cosmological models, the WHIM should be cooler at higher redshift, implying a lower ionization state of the gas (i.e. lower optical depth of high ionization absorbers) and a thermal cutoff further moved to lower energies. Yet, it would be most useful to obtain some constraints on the WHIM properties at high redshift since, when compared with the WHIM detections in the local universe, it would provide contraints on the cosmological models of the evolution of baryons.

To pursue the latter goal we started a detailed investigation of the Lockman Hole, which is one of the fields where the Galactic absorption is minimum $\left(N_{\mathrm{H}} \sim 5.6 \times 10^{19} \mathrm{~cm}^{-2}\right)$ and where deep X-ray observations have been obtained 
Table 1. Objects in the narrow redshift range $0.78<z<0.81$ located within the Lockman Hole reported in the literature.

\begin{tabular}{|c|c|c|c|c|c|}
\hline Object & $\mathrm{RA}(\mathrm{J} 2000)$ & $\operatorname{Dec}(J 2000)$ & Type & $z$ & Reference \\
\hline RDS 117Q & $10 \mathrm{~h} 53 \mathrm{~m} 48.8 \mathrm{~s}$ & $+57^{\circ} 30^{\prime} 34^{\prime \prime}$ & AGN & 0.780 & Lehmann et al. (2001) \\
\hline [HGG98] 5 & $10 \mathrm{~h} 53 \mathrm{~m} 44.9 \mathrm{~s}$ & $+57^{\circ} 35^{\prime} 15^{\prime \prime}$ & Galaxy & 0.782 & Hasinger et al. (1998b) \\
\hline [HGG98] 8 & $10 \mathrm{~h} 53 \mathrm{~m} 42.3 \mathrm{~s}$ & $+57^{\circ} 35^{\prime} 41^{\prime \prime}$ & Galaxy & 0.782 & Hasinger et al. (1998b) \\
\hline RX J105335.1+572542 & $10 \mathrm{~h} 53 \mathrm{~m} 35.1 \mathrm{~s}$ & $+57^{\circ} 25^{\prime} 42^{\prime \prime}$ & QSO & 0.784 & Mainieri et al. (2002) \\
\hline RX J105303.9+572925 & $10 \mathrm{~h} 53 \mathrm{~m} \mathrm{03.9s}$ & $+57^{\circ} 29^{\prime} 25^{\prime \prime}$ & QSO & 0.788 & Mainieri et al. (2002) \\
\hline [MBH2002] 41 & $10 \mathrm{~h} 53 \mathrm{~m} 05.4 \mathrm{~s}$ & $+57^{\circ} 28^{\prime} 10^{\prime \prime}$ & $\mathrm{X}$-ray source & 0.792 & Mainieri et al. (2002) \\
\hline [FFH2002] 105 & $10 \mathrm{~h} 53 \mathrm{~m} 15.80 \mathrm{~s}$ & $+57^{\circ} 24^{\prime} 50.0^{\prime \prime}$ & $\mathrm{X}$-ray source & 0.8 (phot) & Fadda et al. (2002) \\
\hline LOCK-6 cm J105304+573055 & $10 \mathrm{~h} 53 \mathrm{~m} 04.83 \mathrm{~s}$ & $+57^{\circ} 30^{\prime} 55.9^{\prime \prime}$ & Radio source & 0.805 & Ciliegi et al. (2003) \\
\hline RX J105225.3+572246 & $10 \mathrm{~h} 52 \mathrm{~m} 25.3 \mathrm{~s}$ & $+57^{\circ} 22^{\prime} 46^{\prime \prime}$ & AGN & 0.807 & Mainieri et al. (2002) \\
\hline
\end{tabular}

(Hasinger et al. 1998a, 2001; Hasinger 2003). We have searched for superstructures by analyzing the redshift distribution of sources already identified in this field and found 8 sources in a narrow redshift range at about $z \sim 0.8$, located within a region of about 20 arcmin. We obtained multiobject spectroscopy in the same area and, as discussed in Sect. 2, we have confirmed the presence of a superstructure at $z \sim 0.8$. Then we analyzed an XMM map of the LH in the softest band and, as discussed in Sect. 3, we obtained tight constraints on the possible diffuse emission due to WHIM associated with the large-scale structure. In Sect. 4 we discuss these observational constraints on the evolution of the WHIM with redshift.

\section{Optical observations: Detection of a large-scale structure at $z \sim 0.8$}

An analysis of the redshift distribution of the sources previously identified in the Lockman Hole (most of which are optical counterparts of X-ray sources detected by ROSAT and $\mathrm{XMM}$ ) has revealed the existence of 8 sources in the narrow redshift range $0.780-0.807\left(\sim 74 \mathrm{Mpc}^{1}\right)$. These sources (listed in Table 1) are marked with a circle in Fig. 1 and are located within a region of about 20 arcmin. In the same region we have found another object (marked with a diamond) with a photometrically estimated redshift of $0.8 \pm 0.1$. Note that 4 such objects (distributed along the N-S direction) have redshifts in the narrower interval $0.780-0.784$. This is strongly suggestive of the existence of a large-scale structure at this redshift in this region of the Lockman Hole.

To confirm this we have obtained multi-object spectroscopy of 215 galaxies in the same area. We used the multiobject spectroscopic mode (MOS) of the optical spectrometer DOLORES, at the Telescopio Nazionale Galileo (TNG), with the LR-R grating, which covers the 4470-10360 $\AA$ range at a resolution of $11 \AA$. This spectral range allows the identification of $\mathrm{H} \beta+[\mathrm{OIII}]$ and $[\mathrm{OII}]$ at $z \sim 0.8$. The observations were performed over four nights in March 2003.

\footnotetext{
${ }^{1}$ In this paper we assume a cosmology with $\Omega_{\mathrm{m}}=0.3, \Omega_{\Lambda}=0.7$ and $H_{0}=70 \mathrm{~km} \mathrm{~s}^{-1} \mathrm{Mpc}^{-1}$ and all the distances will be expressed in the comoving rest frame.
}

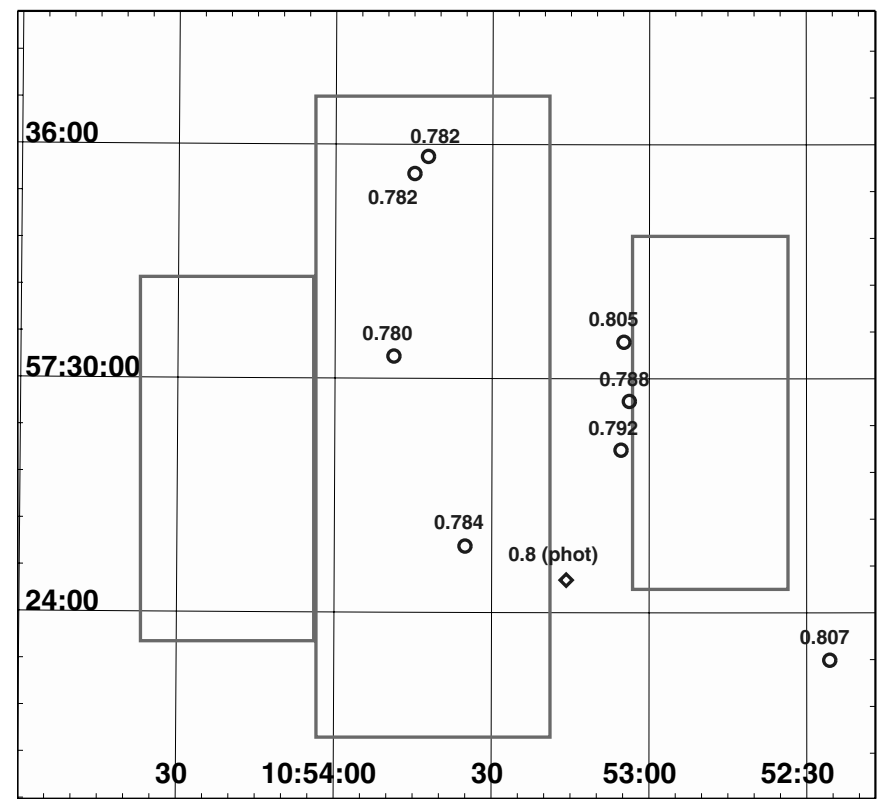

Fig. 1. Spatial distribution of objects reported in the literature in the narrow redshift range $0.78<z<0.81$ within the Lockman Hole (see Table 1). Each object is labelled with its redshift. The object marked with a diamond has a photometric redshift. Boxes indicate the regions covered by our spectroscopic survey with 10 masks.

We selected two samples of galaxies within the subarea of interest of the Lockman Hole. In particular, we selected a shallow sample, made of galaxies in the magnitude range $20.0 \leq R<21.3$, and a deep sample, containing galaxies in the magnitude range $21.3 \leq R<22$. The shallow sample was observed with seven masks and with an integration of $1.5 \mathrm{~h}$ for each mask, while the deep sample was observed with three masks and with an integration of $3 \mathrm{~h}$ per mask. Most of the masks ( 7 of them) were located along the $\mathrm{N}-\mathrm{S}$ direction traced by the 4 sources at $z=0.78$, while three masks were located to the west and to the east to check possible extensions of the putative large scale structure. The location of the various masks is shown by the boxes in Fig. 1.

The MOS spectra were reduced following the standard techniques (dark and bias subtraction, flat fielding, wavelength calibration). The deep sample was observed using a dithering 

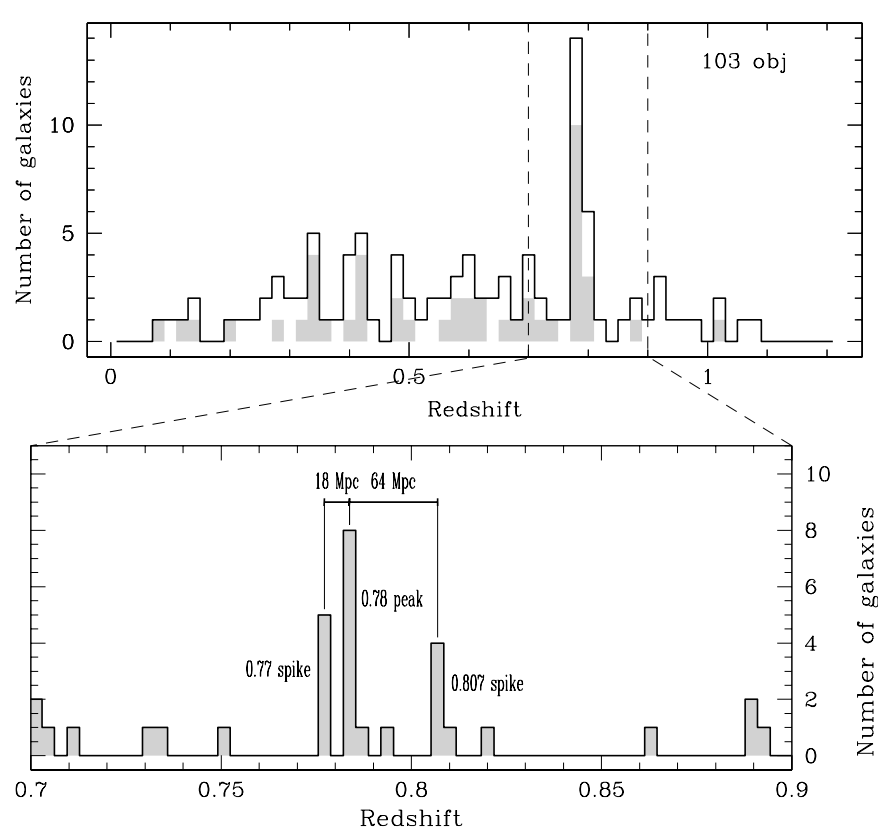

Fig. 2. Upper panel: redshift distribution of the objects for which we have obtained high quality (shaded histogram) and low quality (hollow histogram) spectroscopic redshift measurements. Lower panel: zoom of the redshift distribution around the three main concentrations of objects.

of 6 arcsec along the slit axis to enable a better subtraction of sky lines and an easier detection of the weak emission lines. We observed 215 sources in total. We measured redshifts for 103 objects (see Appendix A for the catalog) with typical random errors of \pm 0.002 , as inferred by the uncertainty in the Gaussian fit to the emission lines ${ }^{2}$. For 47 sources the redshift could be determined unambiguously by the detection of two or more emission lines. We unambiguously assigned the redshift also in the case of detection of only one strong emission line identified as the [OII] line at high redshift ${ }^{3}$ (in Appendix A the quality of these redshifts is marked as "high"). For another 56 objects the redshift determination was less secure, based on line detections with low signal to noise ratio (in Appendix A the quality of these redshifts is marked as "low"). For 112 sources the spectrum was too weak and without bright lines, and we could not recover any information on their redshifts.

The redshift distribution is shown in the top panel of Fig. 2, where a prominent peak $(6 \sigma$ significant with respect to the mean histogram level) is seen at the expected redshift of $z=$ 0.78 , demonstrating beyond any doubt the presence of a largescale structure at this redshift in this region. As for the archival objects, this spike shows two distinct sub-concentrations, one at $z \sim 0.78$ and one at $z \sim 0.807$. The former is further split into a main peak at $z \sim 0.784$ and a nearby spike at $z \sim 0.776$,

\footnotetext{
2 The only source of possible systematic errors is spectral calibration. However, such systematic errors are smaller than the estimated random errors.

${ }^{3}$ Alternatively it would be the $\mathrm{H} \alpha$ of local objects $(z \sim 0)$, but in the latter case the objects should be extended and bright, unlike the ones in our samples.
}

as shown in the bottom panel. Figure 3 shows the distribution projected on the sky of the sources for which the redshift could be determined, and in particular for those in the redshift spikes. Although this redshift survey is not complete, our data suggest that these structures at slightly different redshifts tend also to be distributed in different regions of the sky. In particular, galaxies on the red tail of the main spike, and specifically at $z \sim 0.776$ are located in the southern part of the field, while galaxies in the main spike at $z \sim 0.784$ are preferentially distributed in the northern part. The distribution of the galaxies in the farther spike at $z \sim 0.807$ overlaps with the previous two, but the presence of another source at 0.807 (the one located most to the west in Fig. 3), previously identified by Lehmann et al. (2001), suggests that this substructure extends towards the west. We have tentatively encircled the three main substructures with three ellipses in Fig. 3. On the whole the superstructure outlined by our survey and the archival objects cover a region of $\sim 7.5 \mathrm{Mpc}$ (at the mean redshift $z=0.791$ ). This could be considered as a lower limit to the dimension of the structure because its size is limited by the extent of our observations. However, we note that other spectroscopic surveys covering the entire XMM and ROSAT-HRI fields have not found additional objects in the narrow redshift range outside the area considered by us, suggesting that the large-scale structure is not further extended at least in the XMM and ROSAT-HRI fields.

\section{X-ray data: Constraints on the WHIM emission}

Once having demonstrated that large-scale structures at high redshift exist within the Lockman Hole, we then investigated whether the associated WHIM emission could be detected. Such an investigation requires sensitive maps at soft energies $E<0.5 \mathrm{keV}$, both because of the low temperatures typical of WHIM and because of a significant Doppler shift of the emission to lower energies. Several deep X-ray observations have been carried out in the Lockman Hole area, most importantly with ROSAT and XMM-Newton (Hasinger et al. 1998a, 2001).

ROSAT maps would have the required field of view and sensitivity at low energies to properly constrain the presence of diffuse warm gas at high redshifts. However, the region that we are investigating is also characterized by a high density of X-ray point sources (a fraction of which belonging to the large-scale structure at $z=0.78$ ) and by a few clusters of galaxies (Hasinger et al. 1998b). After the subtraction of the instrumental background and the point source removal through the procedures described in Zappacosta et al. (2002), we find diffuse emission coincident with the superstructure. However, the relatively extended wings of the ROSAT PSF from the point sources probably contribute significantly to the diffuse emission. Indeed, this apparently extended emission elongated in the N-S direction is also found in the harder maps (R45 and R67). The spectral shape of such extended emission is the same as for the point sources, associating this emission with residual wings of point sources as well as a possible detection of unresolved AGNs associated with the large-scale structure (e.g. Gilli et al. 2003). The observed hardness of the emission is also at variance with what is found in other fields where diffuse emission has a much softer spectrum 


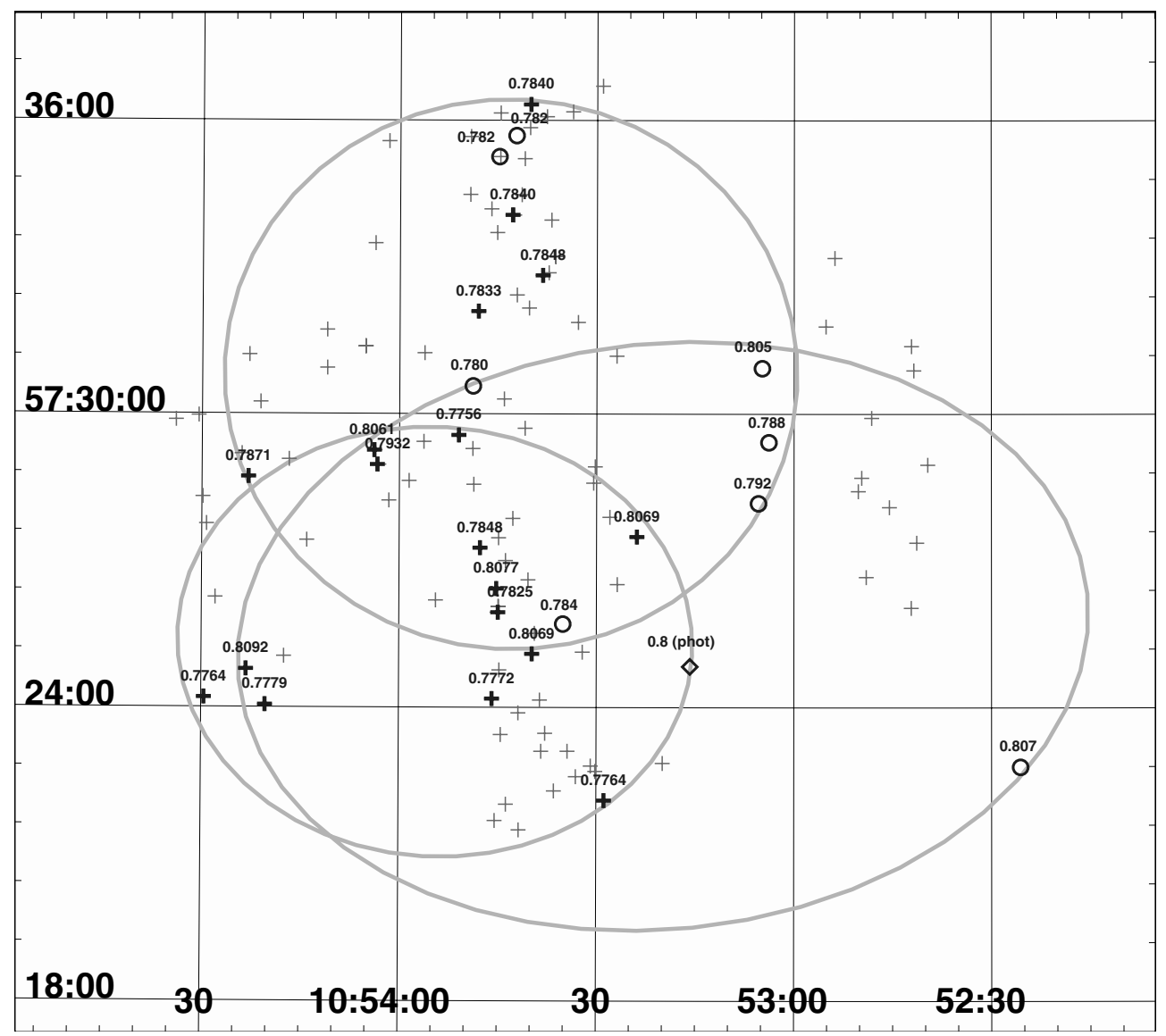

Fig. 3. Spatial distribution of the various spectroscopically identified sources and in particular of those at the redshift corresponding to the spikes at $z \approx 0.8$. Crosses mark the position of all the galaxies with redshifts measured by us (the thick ones are the sources belonging to the superstructure). The circles and the diamond are the archival objects at the same redshift of the spike (as in Fig. 1). For all the galaxies belonging to the superstructure we have reported their measured redshift. The large ellipses tentatively indicate the regions which may be mostly populated by the three subspikes.

(e.g. Zappacosta et al. 2002). The presence of such N-S unresolved AGN emission further suggests the presence of an overdense region of galaxies in this area of the Lockman Hole.

Chandra has a much better angular resolution which allows the removal of the contribution from point sources with a much higher accuracy. However, its sensitivity drops drastically at energies $E<0.5 \mathrm{keV}$, preventing us from studying the level of soft diffuse X-ray emission. The small field of view of ACIS-S (the Chandra chip which has higher sensitivity in the soft band than ACIS-I) is also problematic for the detection of extended emission.

XMM has an appropriate compromise between angular resolution, good sensitivity in the softest X-ray band at $E<$ $0.5 \mathrm{keV}$ and extension of the field of view. The Lockman Hole has been the subject of deep observations with XMM (Hasinger et al. 2001; Hasinger 2003). In particular, the 100 ks observation obtained by Hasinger et al. (2001) was performed with the "thin" filter, which allows the detection of photons down to $0.2 \mathrm{keV}$. An additional $800 \mathrm{ks}$ of integration (Hasinger 2003) were obtained with the "medium" filter, which absorbs photons with $E<0.5 \mathrm{keV}$. The latter observation cannot be used to constrain the WHIM emission because of its energy cutoff at $0.5 \mathrm{keV}$, however it can be efficiently used to subtract spurious contribution to the diffuse emission by hard sources, as we shall discuss later. In the following we will focus on the analysis of the XMM data taken with the "thin" filter.

The major difficulty in using the soft energy band is the presence of the electronic noise that dominates the emission (Lumb et al. 2002; Read \& Ponman 2003). The spectrum of the electronic noise is very stable, and its statistical noise consists of a number of small-amplitude events occurring every frame exposure. There are no fluctuations in a number of events, so the removal of these has no major influence on the detection statistics for the X-ray emission. Electronic noise has a similar spatial distribution for the same detector read-out mode, but its spectrum varies as a function of frame time and is subject to an energy offset on the $10 \mathrm{eV}$ scale for each individual pixel. This energy offset applies to all events, resulting in a decrease of the energy resolution for an extended source. Investigations of the electronic noise by the EPIC calibration team at the MaxPlanck Institut für Extraterrestrische Physik showed that it is possible, by using the shape of the electronic noise, to determine the energy offset in each pixel for each observation and then efficiently and accurately remove the electronic noise from the event lists. The software and processing recipes are made available to the general user as a new task, epreject, within the 


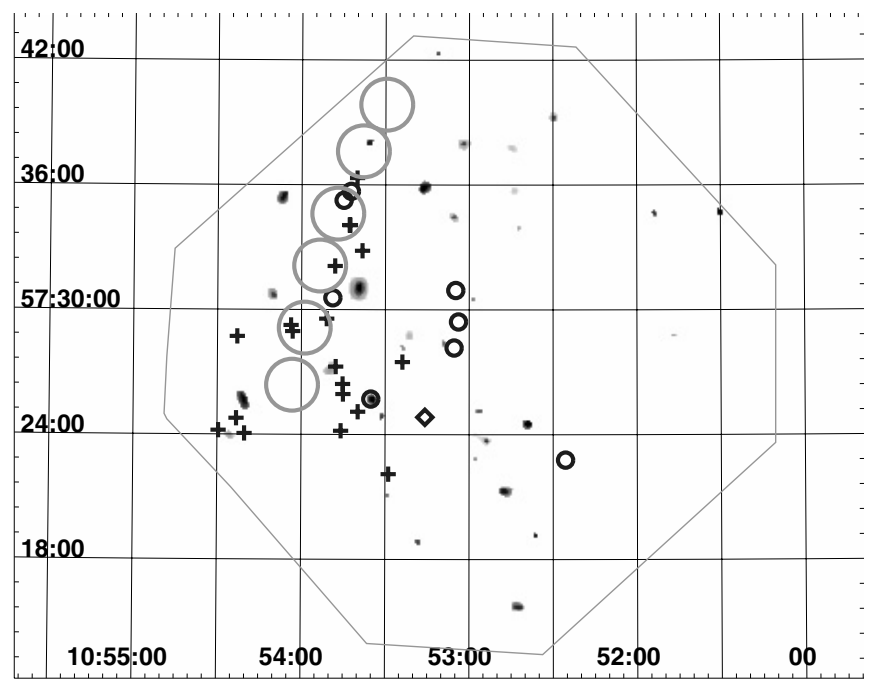

Fig. 4. XMM-Newton EPIC-pn wavelet reconstructed image in the 0.2-0.4 keV band. Crosses, small circles and the diamond are the galaxies belonging to the superstructure detected in the optical as in Fig. 3. Large circles are the regions where we measured the residual soft X-ray flux. The thin line shows the position of the EPIC-pn camera.

SAS 6.0 release. Here we describe the results obtained for a single Lockman Hole pointing as a part of the software testing stage by Konrad Dennerl (see Dennerl et al. (2004) and the epreject task description). For further details of the noise removal and a discussion of associated uncertainties see the epreject task description. We selected the longest EPIC-pn observation, made with the "thin" filter and detector full frame mode (for a description of EPIC-pn see Strüder et al. 2001), which yields $35 \mathrm{ks}$ of useful exposure. For the purposes of our analysis, a subtraction of out-of-time events (OOTE) is not necessary, given the position of bright sources with respect to the superstructure and the orientation of the CCDs in the selected observation. The sensitivity reached by this observation is good enough to set relatively tight constraints on the presence of diffuse soft X-ray emission in the region of the Lockman Hole.

The image in the softest available band $0.2-0.4 \mathrm{keV}$ was extracted and processed through a variable wavelet filter detection algorithm (Vikhlinin et al. 1998) to identify both point and extended sources (see Zappacosta et al. 2002 for details). We set the wavelet peak detection threshold to $4 \sigma$, and followed the extension of the detected flux down to $1.7 \sigma$. We performed ten iterations at wavelet kernel scales ranging from $4^{\prime \prime}$ to $4^{\prime}$. We started from the smallest scale and removed the detected sources prior to proceeding with the next larger scale. "Point sources" were identified as sources detected with kernels of size 4 " (central part of the field of view) and 8 " (outer region, where the PSF is larger). The resulting wavelet map (the sum of all wavelet orders) is shown in Fig. 4. Several point sources are detected as well as a few known clusters. However, we do not find any evidence for diffuse extended emission down to a limit of $2 \sigma$ of confidence from the high order wavelet maps, particularly in the region where the large-scale structure at $z=0.78$ has been detected.
To place limits on the presence of a soft diffuse component associated with the optical structure identified above, we have selected a number of circles along the superstructure traced by galaxies (see in Fig. 4 the big circles). We masked out from the final analysis all the parts where X-ray emission has been detected in the $0.5-2 \mathrm{keV}$ and in the $2-7.5 \mathrm{keV}$ bands in the full $\sim 800 \mathrm{ks}$ XMM-Newton exposure on the Lockman Hole, obtained with the "medium" filter; in this way we could remove instrumental scattering effects, two clusters located near the superstructure, as well as the contribution by unresolved AGNs (which have a much harder spectrum than the WHIM). We then used the $0.2-0.4 \mathrm{keV}$ band to extract the counts from the observation with the thin filter. We also selected two additional regions, closer to the center of the pointing and at the edge of the CCD to estimate both the level of the foreground and background emission close to the position of our measurement and to estimate the possible contribution of the induced background due to the soft protons. To do that, we make a reasonable assumption that the sky components and X-ray foreground components (such as the Local Hot Bubble, LHB) have a flat distribution on the sky, at least within the XMM field of view, and thus are vignetted by the telescope, while the induced background components have a flat distribution of their intensity over the detector (e.g. Lumb et al. 2002).

After subtraction of these in-field estimated background components, the residual soft X-ray flux is $F_{0.2-0.4 \mathrm{keV}}=1.5 \pm$ $1.4 \times 10^{-16} \mathrm{erg} \mathrm{s}^{-1} \mathrm{~cm}^{-2} \operatorname{arcmin}^{-2}$, that increases by $\sim 30 \%$ after the correction for HI absorption. This is a very marginal detection and could be explained by statistical fluctuations around the zero value. Therefore, we consider such a flux as an upper limit to the soft diffuse X-ray emission in this region. It should be noted that the Lockman Hole is the clearest window for this kind of X-ray study, being the region with the lowest Galactic hydrogen column density. This means that a superstructure, as the one we have found in the optical, should clearly show X-ray diffuse emission due to the collapsed gas in the dark matter potential well traced by the galaxies, unless the gas temperature is very low. This issue will be discussed in more detail in the next section.

\section{Temperature of the WHIM at $z \sim 0.8$}

In this section we discuss the upper limit obtained for the diffuse X-ray emission and estimate whether it is compatible with the predictions of the cosmological models and/or whether constraints on the latter can be inferred.

In the local universe $(z<1)$ various detections of emission by WHIM indicate temperatures ranging from $\sim 1 \mathrm{keV}$ down to about $\sim 0.2 \mathrm{keV}$ (Wang et al. 1997; Zappacosta et al. 2002, 2005; Sołtan et al. 2002; Kaastra et al. 2003; Finoguenov et al. 2003). The lower temperature limit could be ascribed to observational issues, since the bulk of the emission from gas with temperatures below $0.1 \mathrm{keV}$ is absorbed even in regions of low Galactic $N_{\mathrm{H}}$. Moreover, low oxygen abundance characteristic of the WHIM (Cen \& Ostriker 1999; Finoguenov et al. 2003) precludes WHIM detection through the OVII emission lines. The situation will change, however, with advent of microcalorimeters with "large grasp". The alternative technique 
of using the OVII and OVIII X-ray absorption lines allowed the detection of Local WHIM at much lower temperatures (Nicastro et al. 2002). For a homogeneous comparison, here we focus on the properties of the WHIM detected in emission.

We take as a reference for the local universe the WHIM emission from the Sculptor supercluster $(z=0.1)$ reported in Zappacosta et al. (2005). In this region the median temperature detected for the WHIM is about $T \sim 0.4 \mathrm{keV}$ (extending up to $0.5 \mathrm{keV}$ and also to $<0.3 \mathrm{keV}$ ). The minimum average WHIM emission (i.e. assuming the maximum possible subtraction of the LHB contribution) is $\sim 240 \times 10^{-6} \mathrm{cts} \mathrm{s}^{-1} \mathrm{arcmin}^{-2}$ in the $0.14-0.28 \mathrm{keV}$ ROSAT band, corresponding to a flux $F_{0.2-0.4 \mathrm{keV}}=9 \times 10^{-16} \mathrm{erg} \mathrm{s}^{-1} \mathrm{~cm}^{-2} \operatorname{arcmin}^{-2}$. We assume that these values are roughly representative of the emission by WHIM in the superstructures of the local universe, however we will show that our conclusions are not critically dependent on such assumptions. If we move such a medium to a redshift of 0.8 , in the absence of evolution its density will increase proportionally to $(1+z)^{3}$ due to the Hubble flow, and more precisely by a factor $[(1+0.8) /(1+0.1)]^{3}=4.4$. However, we also have to include the intrinsic density evolution expected for the WHIM. From Davé et al. (2001, therein Fig. 4) we can estimate that the WHIM at high redshift $(z \sim 1)$ should be denser by a factor of $\sim 1.5$ than locally ${ }^{4}$. When combined with the effect of the Hubble flow, the density increases by a factor $n_{0.8} / n_{0.1}=6.6$. The thermal emission increases proportionally to $n^{2}$, therefore from $z=0.1$ to $z=0.8$ the WHIM emissivity is expected to increase by a factor $\epsilon_{0.8} / \epsilon_{0.1}=\left(n_{0.8} / n_{0.1}\right)^{2}=43.6$. Since we are observing (or constraining) the surface brightness, we also have to account for a cosmological dimming proportional to $(1+z)^{4}$, i.e. $[(1+0.8) /(1+0.1)]^{4}=7.2$. Assuming that the temperature does not change (our working hypothesis) the spectral shape remains unchanged, but the redshift moves the thermal spectrum to lower energies, certainly not enough to push the thermal cutoff in our $0.2-0.4 \mathrm{keV}$ band, but the consequent K-correction still changes the observed flux significantly, and more specifically by a factor of 1.8. Summarizing all of these effects, in the absence of temperature evolution the surface brightness expected for WHIM associated with a superstructure at $z=0.8$ relative to a superstructure at $z=0.1$ is

$\frac{S_{0.2-0.4 \mathrm{keV}}(0.8)}{S_{0.2-0.4 \mathrm{keV}}(0.1)}=\left(\frac{n_{0.8}}{n_{0.1}}\right)^{2}\left(\frac{1+0.8}{1+0.1}\right)^{-4}(1.8)^{-1}=3.4$.

Combined with the surface brightness of the WHIM observed in the Sculptor supercluster, we obtain an expected surface brightness for the WHIM at $z=0.8$ of

$$
S_{0.2-0.4 \mathrm{keV}}(0.8) \approx 3.1 \times 10^{-15} \mathrm{erg} \mathrm{s}^{-1} \mathrm{~cm}^{-2} \operatorname{arcmin}^{-2}
$$

which is roughly an order of magnitude more (more than a factor of 8) than the upper limit obtained by us for the superstructure in the Lockman Hole.

\footnotetext{
${ }^{4}$ These predictions refer to the bulk of the WHIM that, having on average lower densities than the WHIM in supercluster environment, should not be detectable with the current instruments. It is possible that the denser WHIM phase in the supercluster environment evolves in a different way. We are not aware of theoretical works exploring the evolution of this dense WHIM phase.
}

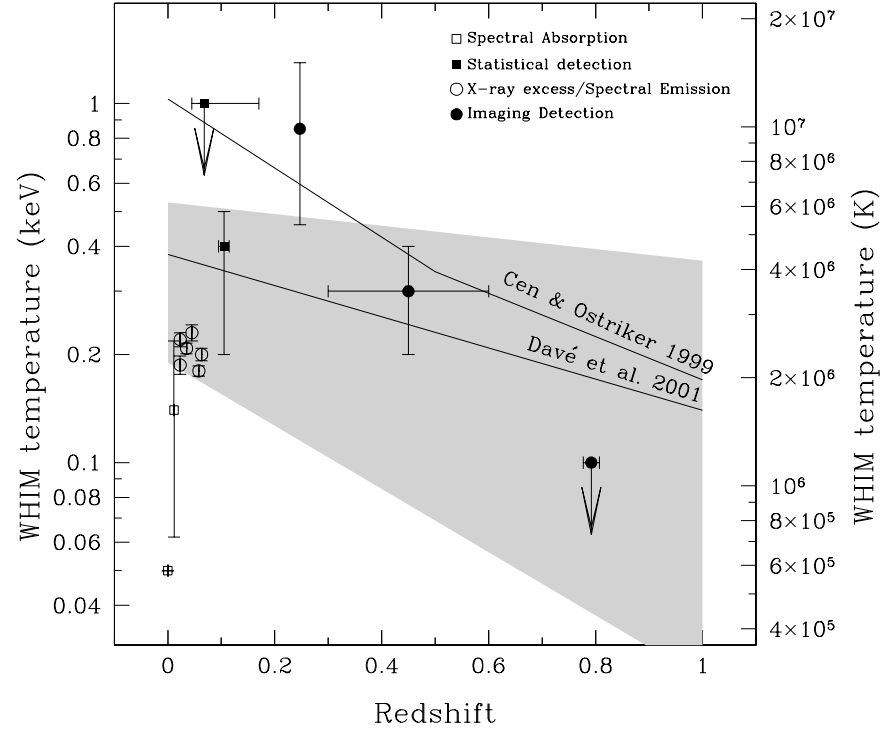

Fig. 5. Observed WHIM temperatures as a function of the redshift taken from the references reported in Table 2. The solid lines are the predictions of Eq. (4) in Cen \& Ostriker (1999) and Davé et al. (2001). The shaded region shows the interval of temperatures which includes 50\% of the WHIM as reported in Fig. 5 of Davé et al. (2001).

One possibility could be that filaments are more rare at high redshift, and that none is present in our field. However, cosmological simulations predict that filamentary structures should already be formed by $z \sim 1$, and that super-structures (traced by overdensity of galaxies) are the locations where filaments are most likely to be present. Since we are clearly investigating one such super-structure (as demonstrated by the galaxy overdensity), the filaments expected by cosmological models at such redshifts are much more likely to be present in our region than in the field. Another, more likely possibility is that baryonic filaments are indeed present in this large-scale structure, but that their temperature at $z=0.8$ is significantly lower than in the local Universe $(z \leq 0.1)$, making them undetectable. Indeed a lower temperature moves the thermal cutoff of the spectrum to lower energies and specifically below the band $0.2-0.4 \mathrm{keV}$ observed by us. We have calculated that the maximum allowed temperature at $z=0.8$, which would make the observed flux consistent with our upper limit in the $0.2-0.4 \mathrm{keV}$ band, is about $0.07-0.1 \mathrm{keV}$. It is important to note that this temperature limit is weakly sensitive on the density and emissivity assumptions discussed above. Indeed, the important result is that the expected flux is so much higher than our upper limit that the temperature must be low enough to move the thermal cutoff below the $0.2-0.4 \mathrm{keV}$ band.

\section{The thermal history of the WHIM}

A summary of the constraints on the observed WHIM temperatures as a function of redshift is given in Fig. 5. We include results by other works listed in Table 2 (both emission and absorption detections). The points give either the median value or the best fitting value. As expected, imaging and statistical measurements, which tend to detect the brightest WHIM emissions, obtain higher temperature values. This is a consequence 
Table 2. WHIM temperatures (sorted by redsfhift) measured so far with different methods by various authors.

\begin{tabular}{lrrrrr}
\hline \hline Object & $z$ & Object type & Method & $k T(\mathrm{keV})$ & Reference \\
\hline PKS 2155-304 & $0^{a}$ & QSO & Spectral absorption & $\sim 0.043$ & Nicastro et al. (2002) \\
Mkn 421 & $0.0116^{a}$ & QSO & Spectral absorption & $0.06 \div 0.22$ & Nicastro (2003) \\
Coma & 0.0231 & Cluster of Galaxies & Soft excess & $0.22 \pm 0.01^{b}$ & Finoguenov et al. (2003) \\
Coma & 0.0231 & Cluster of Galaxies & Soft excess & $0.187 \pm 0.011$ & Kaastra et al. (2003) \\
Abell 2052 & 0.035 & Cluster of Galaxies & Soft excess & $0.208 \pm 0.007$ & Kaastra et al. (2003) \\
MKW 3s & 0.045 & Cluster of Galaxies & Soft excess & $0.23 \pm 0.012$ & Kaastra et al. (2003) \\
Sersic 159 03 & 0.058 & Cluster of Galaxies & Soft excess & $0.18 \pm 0.006$ & Kaastra et al. (2003) \\
Abell 1795 & 0.0631 & Cluster of Galaxies & Soft excess & $0.2 \pm 0.008$ & Kaastra et al. (2003) \\
Abell Clusters & $0.068_{-0.023}^{+0.102 c}$ & Clusters of Galaxies & Statistical & $<1$ & Sołtan et al. (2002) \\
Sculptor Scl & $0.105 \pm 0.01$ & Supercluster & Statistical & $0.4_{-0.2}^{+0.1 c}$ & Zappacosta et al. (2005) \\
Abell 2125 & 0.247 & Cluster of Galaxies & Imaging & $0.85_{-0.39}^{+0.45 d}$ & Wang et al. (1997) \\
Warwick field & $0.45 \pm 0.15$ & Field & Imaging & $0.3 \pm 0.15^{c}$ & Zappacosta et al. (2002) \\
Lockman Hole & $0.791 \pm 0.016$ & Superstructure & Imaging & $<0.1$ & This paper \\
\hline
\end{tabular}

${ }^{a}$ Redshift of the absorption system.

${ }^{b} 1 \sigma$ error-bars.

${ }^{c}$ Median value.

${ }^{d} 90 \%$ level of confidence.

of the WHIM emissivity per unit energy being proportional to the temperature ${ }^{5}$. On the contrary, absorption systems sample random regions of the WHIM, including the very low temperature regions. Soft excess around clusters also tend to be biased against high temperatures, because the latter would be confused with the cluster emission itself. Moreover the WHIM origin of cluster soft excesses have recently been partly brought into question by cosmological simulations (Mittaz et al. 2004; Cheng et al. 2004).

An homogeneous comparison should be limited to detections (and upper limits) made with the same technique. In this paper we have searched for WHIM emission, and therefore our upper limit must be compared with the other detections and upper limits obtained with imaging or statistical detections of WHIM emission (full symbols in Fig. 5). Such measurements indicate that the observed WHIM temperature decreases with redshift (Fig. 5), just as predicted by cosmological models. The number of observational data is small and with large errorbars, however the plot in Fig. 5 is the first attempt at constraining the evolution of the WHIM temperature with redshift, with the currently available data.

It is possible to go beyond this qualitative statement and investigate the quantitative agreement with the model predictions. Cen \& Ostriker (1999) presented in their paper a simple argument that can reproduce representative values for the temperature of the WHIM (see also Fig. 5 in Davé et al. 2001). They infer the postshock temperature of a cosmic gas collapsing inside a slightly nonlinear large-scale structure of

5 The thermal emissivity per unit energy is related to the temperature $T$ and the density $n$ as $T^{-0.5} n^{2}$ (at energies below the thermal cutoff). On the other hand Davé et al. (2001) showed that the WHIM temperature and density are directly proportional, and therefore $\epsilon \propto T^{1.5}$. size $L$ as $T \propto c_{\mathrm{s}}^{2} \approx K\left(L t_{\mathrm{H}}^{-1}(z)\right)^{2}$, where $c_{\mathrm{s}}$ is the gas sound speed and $t_{\mathrm{H}}(z)$ the Hubble time at the redshift of interest and $K$ is constant (see Eq. (4) in Cen \& Ostriker 1999). Such a simple estimate is shown to correctly reproduce the results from numerical simulations (Cen \& Ostriker 1999; Davé et al. 2001). The same approach was used by Davé et al. (2001) who obtain similar results. In Fig. 5 we show the trend of the WHIM temperature as a function of redshift obtained by Cen \& Ostriker (1999) and Davé et al. (2001) by using the argument discussed above (the shaded area indicates the interval of temperatures which includes $\sim 50 \%$ of the WHIM in the Davé et al. (2001) distributions). Although the uncertainties of the theoretical models are large, both predictions fit reasonably well the data points, and in particular the temperatures inferred from the imaging and statistical methods.

\section{Conclusions}

We have identified a large-scale structure of galaxies in the Lockman Hole at redshift $z \sim 0.79 \pm 0.015$ by means of an optical spectroscopic survey. The superstructure extends over a region of more than $7.5 \mathrm{Mpc}$ (in projection) and is structured in three sub-concentrations at median redshifts of $0.776,0.784$ and 0.806 . In this superstructure the WHIM predicted by cosmological models should have already formed. By analysing ROSAT and XMM pointings we could set a tight upper limit on the WHIM emission associated with the superstructure. From this flux limit we could estimate an upper limit of $\sim 0.1 \mathrm{keV}$ on the WHIM temperature at $z \sim 0.8$. The combination of this tight upper limit with other previous WHIM temperature measurements (at lower redshifts) strongly suggests that the WHIM temperature must be rapidly decreasing with redshift, as expected by the cosmological models. The agreement of 
the redshift distribution of the observed WHIM temperatures with the cosmological predictions (Cen \& Ostriker 1999; Davé et al. 2001) is reasonably good even from a quantitative point of view. However further work is required to improve the statistics of the WHIM temperature measurements (or constraints) at high redshift.

Acknowledgements. The paper is based on observations obtained with XMM-Newton, an ESA science mission with instruments and contributions directly funded by ESA Member States and the USA (NASA). The XMM-Newton project is supported by the Bundesministerium für Bildung und Forschung/Deutsches Zentrum für Luft- und Raumfahrt (BMFT/DLR), the Max-Planck Society and the Heidenhain-Stiftung, and also by PPARC, CEA, CNES, and ASI. This work was partially supported by the Italian Ministry of Research (MIUR) and by the Italian Institute of Astrophysics (INAF). We thank G. Hasinger, H. Boehringer, X. Barcons and A. Fabian for letting us use the Lockman Hole image to remove point source contamination and Konrad Dennerl for his assistance with XMM data analysis. A.F. acknowledges support from BMBF/DLR under grant 50 OR 0207 and MPG.

\section{Appendix A: Catalogue of objects}

\begin{tabular}{|c|c|c|c|c|}
\hline Id & RA (J2000) & Dec (J2000) & $z$ & Quality \\
\hline 1 & $10^{\mathrm{h}} 53^{\mathrm{m}} 41.77^{\mathrm{s}}$ & $+57^{\circ} 21^{\prime} 29.56^{\prime \prime}$ & 0.281 & low \\
\hline 2 & $10^{\mathrm{h}} 53^{\mathrm{m}} 45.41^{\mathrm{s}}$ & $+57^{\circ} 21^{\prime} 40.96^{\prime \prime}$ & 0.085 & high \\
\hline 3 & $10^{\mathrm{h}} 53^{\mathrm{m}} 43.71^{\mathrm{s}}$ & $+57^{\circ} 22^{\prime} 01.01^{\prime \prime}$ & 0.608 & low \\
\hline 4 & $10^{\mathrm{h}} 53^{\mathrm{m}} 28.84^{\mathrm{s}}$ & $+57^{\circ} 22^{\prime} 05.78^{\prime \prime}$ & 0.776 & low \\
\hline 5 & $10^{\mathrm{h}} 53^{\mathrm{m}} 36.44^{\mathrm{s}}$ & $+57^{\circ} 22^{\prime} 17.45^{\prime \prime}$ & 0.482 & low \\
\hline 6 & $10^{\mathrm{h}} 53^{\mathrm{m}} 33.12^{\mathrm{s}}$ & $+57^{\circ} 22^{\prime} 34.99^{\prime \prime}$ & 0.735 & high \\
\hline 7 & $10^{\mathrm{h}} 53^{\mathrm{m}} 30.17^{\mathrm{s}}$ & $+57^{\circ} 22^{\prime} 41.48^{\prime \prime}$ & 0.863 & low \\
\hline 8 & $10^{\mathrm{h}} 53^{\mathrm{m}} 30.86^{\mathrm{s}}$ & $+57^{\circ} 22^{\prime} 48.10^{\prime \prime}$ & 0.634 & low \\
\hline 9 & $10^{\mathrm{h}} 53^{\mathrm{m}} 19.95^{\mathrm{s}}$ & $+57^{\circ} 22^{\prime} 51.48^{\prime \prime}$ & 0.528 & low \\
\hline 10 & $10^{\mathrm{h}} 53^{\mathrm{m}} 34.39^{\mathrm{s}}$ & $+57^{\circ} 23^{\prime} 06.16^{\prime \prime}$ & 0.405 & low \\
\hline 11 & $10^{\mathrm{h}} 53^{\mathrm{m}} 38.38^{\mathrm{s}}$ & $+57^{\circ} 23^{\prime} 06.04^{\prime \prime}$ & 0.419 & high \\
\hline 12 & $10^{\mathrm{h}} 53^{\mathrm{m}} 44.53^{\mathrm{s}}$ & $+57^{\circ} 23^{\prime} 26.43^{\prime \prime}$ & 1.017 & high \\
\hline 13 & $10^{\mathrm{h}} 53^{\mathrm{m}} 37.79^{\mathrm{s}}$ & $+57^{\circ} 23^{\prime} 28.09^{\prime \prime}$ & 0.436 & low \\
\hline 14 & $10^{\mathrm{h}} 53^{\mathrm{m}} 41.86^{\mathrm{s}}$ & $+57^{\circ} 23^{\prime} 53.08^{\prime \prime}$ & 0.892 & low \\
\hline 15 & $10^{\mathrm{h}} 53^{\mathrm{m}} 45.87^{\mathrm{s}}$ & $+57^{\circ} 24^{\prime} 10.48^{\prime \prime}$ & 0.777 & high \\
\hline 16 & $10^{\mathrm{h}} 53^{\mathrm{m}} 38.60^{\mathrm{s}}$ & $+57^{\circ} 24^{\prime} 08.85^{\prime \prime}$ & 0.484 & high \\
\hline 17 & $10^{\mathrm{h}} 53^{\mathrm{m}} 47.71^{\mathrm{s}}$ & $+57^{\circ} 27^{\prime} 15.48^{\prime \prime}$ & 0.785 & high \\
\hline 18 & $10^{\mathrm{h}} 53^{\mathrm{m}} 43.83^{\mathrm{s}}$ & $+57^{\circ} 26^{\prime} 59.82^{\prime \prime}$ & 0.729 & low \\
\hline 19 & $10^{\mathrm{h}} 53^{\mathrm{m}} 54.44^{\mathrm{s}}$ & $+57^{\circ} 26^{\prime} 11.21^{\prime \prime}$ & 0.644 & low \\
\hline 20 & $10^{\mathrm{h}} 53^{\mathrm{m}} 40.40^{\mathrm{s}}$ & $+57^{\circ} 26^{\prime} 36.23^{\prime \prime}$ & 0.821 & low \\
\hline 21 & $10^{\mathrm{h}} 53^{\mathrm{m}} 45.22^{\mathrm{s}}$ & $+57^{\circ} 26^{\prime} 25.45^{\prime \prime}$ & 0.808 & high \\
\hline 22 & $10^{\mathrm{h}} 53^{\mathrm{m}} 26.84^{\mathrm{s}}$ & $+57^{\circ} 26^{\prime} 30.70^{\prime \prime}$ & 0.354 & high \\
\hline 23 & $10^{\mathrm{h}} 53^{\mathrm{m}} 45.09^{\mathrm{s}}$ & $+57^{\circ} 25^{\prime} 56.43^{\prime \prime}$ & 0.783 & high \\
\hline 24 & $10^{\mathrm{h}} 53^{\mathrm{m}} 23.87^{\mathrm{s}}$ & $+57^{\circ} 27^{\prime} 29.10^{\prime \prime}$ & 0.807 & low \\
\hline 25 & $10^{\mathrm{h}} 53^{\mathrm{m}} 44.91^{\mathrm{s}}$ & $+57^{\circ} 26^{\prime} 03.48^{\prime \prime}$ & 0.619 & high \\
\hline
\end{tabular}

\begin{tabular}{|c|c|c|c|c|}
\hline Id & RA (J2000) & Dec (J2000) & $z$ & Quality \\
\hline 26 & $10^{\mathrm{h}} 53^{\mathrm{m}} 39.80^{\mathrm{s}}$ & $+57^{\circ} 25^{\prime} 05.50^{\prime \prime}$ & 0.807 & high \\
\hline 27 & $10^{\mathrm{h}} 53^{\mathrm{m}} 39.41^{\mathrm{s}}$ & $+57^{\circ} 25^{\prime} 30.62^{\prime \prime}$ & 0.335 & high \\
\hline 28 & $10^{\mathrm{h}} 53^{\mathrm{m}} 44.89^{\mathrm{s}}$ & $+57^{\circ} 27^{\prime} 27.74^{\prime \prime}$ & 0.594 & high \\
\hline 29 & $10^{\mathrm{h}} 53^{\mathrm{m}} 32.13^{\mathrm{s}}$ & $+57^{\circ} 25^{\prime} 07.74^{\prime \prime}$ & 0.479 & high \\
\hline 30 & $10^{\mathrm{h}} 53^{\mathrm{m}} 44.77^{\mathrm{s}}$ & $+57^{\circ} 24^{\prime} 45.67^{\prime \prime}$ & 0.917 & low \\
\hline 31 & $10^{\mathrm{h}} 53^{\mathrm{m}} 27.98^{\mathrm{s}}$ & $+57^{\circ} 27^{\prime} 53.20^{\prime \prime}$ & 0.146 & low \\
\hline 32 & $10^{\mathrm{h}} 53^{\mathrm{m}} 42.73^{\mathrm{s}}$ & $+57^{\circ} 27^{\prime} 51.53^{\prime \prime}$ & 0.684 & high \\
\hline 33 & $10^{\mathrm{h}} 53^{\mathrm{m}} 48.68^{\mathrm{s}}$ & $+57^{\circ} 28^{\prime} 33.26^{\prime \prime}$ & 0.279 & low \\
\hline 34 & $10^{\mathrm{h}} 53^{\mathrm{m}} 58.52^{\mathrm{s}}$ & $+57^{\circ} 28^{\prime} 37.64^{\prime \prime}$ & 0.751 & low \\
\hline 35 & $10^{\mathrm{h}} 53^{\mathrm{m}} 30.46^{\mathrm{s}}$ & $+57^{\circ} 28^{\prime} 35.05^{\prime \prime}$ & 0.531 & low \\
\hline 36 & $10^{\mathrm{h}} 53^{\mathrm{m}} 30.22^{\mathrm{s}}$ & $+57^{\circ} 28^{\prime} 55.08^{\prime \prime}$ & 1.011 & low \\
\hline 37 & $10^{\mathrm{h}} 53^{\mathrm{m}} 48.84^{\mathrm{s}}$ & $+57^{\circ} 29^{\prime} 17.08^{\prime \prime}$ & 0.889 & high \\
\hline 38 & $10^{\mathrm{h}} 53^{\mathrm{m}} 56.29^{\mathrm{s}}$ & $+57^{\circ} 29^{\prime} 25.65^{\prime \prime}$ & 0.580 & high \\
\hline 39 & $10^{\mathrm{h}} 53^{\mathrm{m}} 50.98^{\mathrm{s}}$ & $+57^{\circ} 29^{\prime} 33.65^{\prime \prime}$ & 0.776 & low \\
\hline 40 & $10^{\mathrm{h}} 53^{\mathrm{m}} 40.91^{\mathrm{s}}$ & $+57^{\circ} 29^{\prime} 42.15^{\prime \prime}$ & 0.919 & low \\
\hline 41 & $10^{\mathrm{h}} 53^{\mathrm{m}} 44.08^{\mathrm{s}}$ & $+57^{\circ} 30^{\prime} 17.99^{\prime \prime}$ & 0.490 & low \\
\hline 42 & $10^{\mathrm{h}} 53^{\mathrm{m}} 26.96^{\mathrm{s}}$ & $+57^{\circ} 31^{\prime} 10.83^{\prime \prime}$ & 0.377 & low \\
\hline 43 & $10^{\mathrm{h}} 53^{\mathrm{m}} 56.19^{\mathrm{s}}$ & $+57^{\circ} 31^{\prime} 14.36^{\prime \prime}$ & 0.701 & low \\
\hline 44 & $10^{\mathrm{h}} 53^{\mathrm{m}} 32.85^{\mathrm{s}}$ & $+57^{\circ} 31^{\prime} 52.09^{\prime \prime}$ & 0.422 & high \\
\hline 45 & $10^{\mathrm{h}} 53^{\mathrm{m}} 48.02^{\mathrm{s}}$ & $+57^{\circ} 32^{\prime} 05.64^{\prime \prime}$ & 0.783 & low \\
\hline 46 & $10^{\mathrm{h}} 53^{\mathrm{m}} 40.31^{\mathrm{s}}$ & $+57^{\circ} 32^{\prime} 09.61^{\prime \prime}$ & 0.692 & low \\
\hline 47 & $10^{\mathrm{h}} 53^{\mathrm{m}} 42.17^{\mathrm{s}}$ & $+57^{\circ} 32^{\prime} 25.60^{\prime \prime}$ & 0.321 & high \\
\hline 48 & $10^{\mathrm{h}} 53^{\mathrm{m}} 49.23^{\mathrm{s}}$ & $+57^{\circ} 35^{\prime} 39.75^{\prime \prime}$ & 0.231 & low \\
\hline 49 & $10^{\mathrm{h}} 53^{\mathrm{m}} 49.31^{\mathrm{s}}$ & $+57^{\circ} 34^{\prime} 28.74^{\prime \prime}$ & 0.391 & low \\
\hline 50 & $10^{\mathrm{h}} 53^{\mathrm{m}} 40.12^{\mathrm{s}}$ & $+57^{\circ} 36^{\prime} 19.28^{\prime \prime}$ & 0.784 & high \\
\hline 51 & $10^{\mathrm{h}} 53^{\mathrm{m}} 33.68^{\mathrm{s}}$ & $+57^{\circ} 36^{\prime} 10.40^{\prime \prime}$ & 0.500 & high \\
\hline 52 & $10^{\mathrm{h}} 53^{\mathrm{m}} 37.67^{\mathrm{s}}$ & $+57^{\circ} 36^{\prime} 04.33^{\prime \prime}$ & 0.405 & low \\
\hline 53 & $10^{\mathrm{h}} 53^{\mathrm{m}} 40.23^{\mathrm{s}}$ & $+57^{\circ} 35^{\prime} 50.81^{\prime \prime}$ & 0.933 & low \\
\hline 54 & $10^{\mathrm{h}} 53^{\mathrm{m}} 44.73^{\mathrm{s}}$ & $+57^{\circ} 36^{\prime} 08.73^{\prime \prime}$ & 0.591 & low \\
\hline 55 & $10^{\mathrm{h}} 54^{\mathrm{m}} 01.68^{\mathrm{s}}$ & $+57^{\circ} 35^{\prime} 34.23^{\prime \prime}$ & 0.256 & low \\
\hline 56 & $10^{\mathrm{h}} 53^{\mathrm{m}} 41.03^{\mathrm{s}}$ & $+57^{\circ} 35^{\prime} 12.77^{\prime \prime}$ & 0.493 & low \\
\hline 57 & $10^{\mathrm{h}} 53^{\mathrm{m}} 44.76^{\mathrm{s}}$ & $+57^{\circ} 35^{\prime} 15.24^{\prime \prime}$ & 0.093 & low \\
\hline 58 & $10^{\mathrm{h}} 53^{\mathrm{m}} 42.62^{\mathrm{s}}$ & $+57^{\circ} 34^{\prime} 03.58^{\prime \prime}$ & 0.784 & high \\
\hline 59 & $10^{\mathrm{h}} 53^{\mathrm{m}} 41.48^{\mathrm{s}}$ & $+57^{\circ} 34^{\prime} 28.55^{\prime \prime}$ & 0.256 & low \\
\hline 60 & $10^{\mathrm{h}} 53^{\mathrm{m}} 46.09^{\mathrm{s}}$ & $+57^{\circ} 34^{\prime} 11.09^{\prime \prime}$ & 1.084 & low \\
\hline 61 & $10^{\mathrm{h}} 53^{\mathrm{m}} 42.87^{\mathrm{s}}$ & $+57^{\circ} 34^{\prime} 03.80^{\prime \prime}$ & 0.784 & high \\
\hline 62 & $10^{\mathrm{h}} 53^{\mathrm{m}} 36.94^{\mathrm{s}}$ & $+57^{\circ} 33^{\prime} 57.55^{\prime \prime}$ & 0.420 & high \\
\hline 63 & $10^{\mathrm{h}} 53^{\mathrm{m}} 36.32^{\mathrm{s}}$ & $+57^{\circ} 33^{\prime} 13.74^{\prime \prime}$ & 0.706 & high \\
\hline 64 & $10^{\mathrm{h}} 53^{\mathrm{m}} 36.32^{\mathrm{s}}$ & $+57^{\circ} 33^{\prime} 12.91^{\prime \prime}$ & 0.784 & high \\
\hline 65 & $10^{\mathrm{h}} 53^{\mathrm{m}} 45.19^{\mathrm{s}}$ & $+57^{\circ} 33^{\prime} 42.05^{\prime \prime}$ & 0.701 & high \\
\hline 66 & $10^{\mathrm{h}} 54^{\mathrm{m}} 03.72^{\mathrm{s}}$ & $+57^{\circ} 33^{\prime} 29.03^{\prime \prime}$ & 0.662 & low \\
\hline 67 & $10^{\mathrm{h}} 53^{\mathrm{m}} 37.33^{\mathrm{s}}$ & $+57^{\circ} 32^{\prime} 52.90^{\prime \prime}$ & 0.562 & low \\
\hline 68 & $10^{\mathrm{h}} 53^{\mathrm{m}} 38.25^{\mathrm{s}}$ & $+57^{\circ} 32^{\prime} 49.74^{\prime \prime}$ & 0.785 & high \\
\hline 69 & $10^{\mathrm{h}} 53^{\mathrm{m}} 29.13^{\mathrm{s}}$ & $+57^{\circ} 36^{\prime} 41.92^{\prime \prime}$ & 0.427 & high \\
\hline
\end{tabular}




\begin{tabular}{|c|c|c|c|c|}
\hline Id & RA (J2000) & $\operatorname{Dec}(\mathrm{J} 2000)$ & $z$ & Quality \\
\hline 70 & $10^{\mathrm{h}} 52^{\mathrm{m}} 50.23^{\mathrm{s}}$ & $+57^{\circ} 28^{\prime} 25.09^{\prime \prime}$ & 0.536 & low \\
\hline 71 & $10^{\mathrm{h}} 52^{\mathrm{m}} 49.71^{\mathrm{s}}$ & $+57^{\circ} 28^{\prime} 41.56^{\prime \prime}$ & 0.342 & high \\
\hline 72 & $10^{\mathrm{h}} 52^{\mathrm{m}} 39.67^{\mathrm{s}}$ & $+57^{\circ} 28^{\prime} 57.53^{\prime \prime}$ & 0.669 & low \\
\hline 73 & $10^{\mathrm{h}} 52^{\mathrm{m}} 48.20^{\mathrm{s}}$ & $+57^{\circ} 29^{\prime} 54.99^{\prime \prime}$ & 0.291 & low \\
\hline 74 & $10^{\mathrm{h}} 52^{\mathrm{m}} 41.79^{\mathrm{s}}$ & $+57^{\circ} 30^{\prime} 53.26^{\prime \prime}$ & 0.206 & high \\
\hline 75 & $10^{\mathrm{h}} 52^{\mathrm{m}} 42.14^{\mathrm{s}}$ & $+57^{\circ} 31^{\prime} 23.02^{\prime \prime}$ & 0.121 & high \\
\hline 76 & $10^{\mathrm{h}} 52^{\mathrm{m}} 55.15^{\mathrm{s}}$ & $+57^{\circ} 31^{\prime} 47.06^{\prime \prime}$ & 0.343 & low \\
\hline 77 & $10^{\mathrm{h}} 52^{\mathrm{m}} 53.80^{\mathrm{s}}$ & $+57^{\circ} 33^{\prime} 11.11^{\prime \prime}$ & 0.669 & high \\
\hline 78 & $10^{\mathrm{h}} 52^{\mathrm{m}} 45.50^{\mathrm{s}}$ & $+57^{\circ} 28^{\prime} 05.49^{\prime \prime}$ & 0.606 & high \\
\hline 79 & $10^{\mathrm{h}} 52^{\mathrm{m}} 41.35^{\mathrm{s}}$ & $+57^{\circ} 27^{\prime} 21.95^{\prime \prime}$ & 0.552 & high \\
\hline 80 & $10^{\mathrm{h}} 52^{\mathrm{m}} 49.02^{\mathrm{s}}$ & $+57^{\circ} 26^{\prime} 39.76^{\prime \prime}$ & 0.322 & low \\
\hline 81 & $10^{\mathrm{h}} 52^{\mathrm{m}} 42.18^{\mathrm{s}}$ & $+57^{\circ} 26^{\prime} 02.02^{\prime \prime}$ & 0.343 & high \\
\hline 82 & $10^{\mathrm{h}} 54^{\mathrm{m}} 20.31^{\mathrm{s}}$ & $+57^{\circ} 24^{\prime} 02.86^{\prime \prime}$ & 0.778 & low \\
\hline 83 & $10^{\mathrm{h}} 54^{\mathrm{m}} 29.59^{\mathrm{s}}$ & $+57^{\circ} 24^{\prime} 11.82^{\prime \prime}$ & 0.776 & high \\
\hline 84 & $10^{\mathrm{h}} 54^{\mathrm{m}} 23.22^{\mathrm{s}}$ & $+57^{\circ} 24^{\prime} 46.59^{\prime \prime}$ & 0.809 & low \\
\hline 85 & $10^{\mathrm{h}} 54^{\mathrm{m}} 17.46^{\mathrm{s}}$ & $+57^{\circ} 25^{\prime} 02.44^{\prime \prime}$ & 0.138 & high \\
\hline 86 & $10^{\mathrm{h}} 54^{\mathrm{m}} 27.92^{\mathrm{s}}$ & $+57^{\circ} 26^{\prime} 14.55^{\prime \prime}$ & 0.578 & high \\
\hline 87 & $10^{\mathrm{h}} 54^{\mathrm{m}} 14.04^{\mathrm{s}}$ & $+57^{\circ} 27^{\prime} 24.94^{\prime \prime}$ & 0.913 & low \\
\hline 88 & $10^{\mathrm{h}} 54^{\mathrm{m}} 29.28^{\mathrm{s}}$ & $+57^{\circ} 27^{\prime} 44.63^{\prime \prime}$ & 0.306 & low \\
\hline 89 & $10^{\mathrm{h}} 54^{\mathrm{m}} 11.02^{\mathrm{s}}$ & $+57^{\circ} 31^{\prime} 42.79^{\prime \prime}$ & 0.396 & high \\
\hline 90 & $10^{\mathrm{h}} 54^{\mathrm{m}} 22.84^{\mathrm{s}}$ & $+57^{\circ} 31^{\prime} 12.17^{\prime \prime}$ & 0.343 & high \\
\hline 91 & $10^{\mathrm{h}} 54^{\mathrm{m}} 05.16^{\mathrm{s}}$ & $+57^{\circ} 31^{\prime} 20.91^{\prime \prime}$ & 0.977 & low \\
\hline 92 & $10^{\mathrm{h}} 54^{\mathrm{m}} 05.16^{\mathrm{s}}$ & $+57^{\circ} 31^{\prime} 22.56^{\prime \prime}$ & 1.062 & low \\
\hline 93 & $10^{\mathrm{h}} 54^{\mathrm{m}} 10.99^{\mathrm{s}}$ & $+57^{\circ} 30^{\prime} 56.14^{\prime \prime}$ & 0.226 & low \\
\hline 94 & $10^{\mathrm{h}} 54^{\mathrm{m}} 30.51^{\mathrm{s}}$ & $+57^{\circ} 29^{\prime} 57.57^{\prime \prime}$ & 0.619 & high \\
\hline 95 & $10^{\mathrm{h}} 54^{\mathrm{m}} 01.59^{\mathrm{s}}$ & $+57^{\circ} 28^{\prime} 13.58^{\prime \prime}$ & 0.890 & low \\
\hline 96 & $10^{\mathrm{h}} 54^{\mathrm{m}} 03.72^{\mathrm{s}}$ & $+57^{\circ} 29^{\prime} 15.01^{\prime \prime}$ & 0.806 & high \\
\hline 97 & $10^{\mathrm{h}} 54^{\mathrm{m}} 23.93^{\mathrm{s}}$ & $+57^{\circ} 29^{\prime} 10.58^{\prime \prime}$ & 0.410 & low \\
\hline 98 & $10^{\mathrm{h}} 54^{\mathrm{m}} 16.76^{\mathrm{s}}$ & $+57^{\circ} 29^{\prime} 03.93^{\prime \prime}$ & 0.589 & low \\
\hline 99 & $10^{\mathrm{h}} 54^{\mathrm{m}} 03.14^{\mathrm{s}}$ & $+57^{\circ} 28^{\prime} 57.45^{\prime \prime}$ & 0.793 & low \\
\hline 100 & $10^{\mathrm{h}} 54^{\mathrm{m}} 22.93^{\mathrm{s}}$ & $+57^{\circ} 28^{\prime} 42.62^{\prime \prime}$ & 0.787 & high \\
\hline 101 & $10^{\mathrm{h}} 54^{\mathrm{m}} 29.85^{\mathrm{s}}$ & $+57^{\circ} 28^{\prime} 17.79^{\prime \prime}$ & 0.711 & high \\
\hline 102 & $10^{\mathrm{h}} 54^{\mathrm{m}} 21.11^{\mathrm{s}}$ & $+57^{\circ} 30^{\prime} 14.17^{\prime \prime}$ & 0.965 & low \\
\hline 103 & $10^{\mathrm{h}} 54^{\mathrm{m}} 33.97^{\mathrm{s}}$ & $+57^{\circ} 29^{\prime} 52.14^{\prime \prime}$ & 0.276 & high \\
\hline
\end{tabular}

\section{References}

Cen, R., \& Ostriker, J. P. 1999, ApJ, 514, 1

Cheng, L.-M., Borgani, S., Tozzi, P., et al. 2004 [arXiv: astro-ph/0409707]

Ciliegi, P., Zamorani, G., Hasinger, G., et al. 2003, A\&A, 398, 901 Davé, R., Cen, R., Ostriker, J. P., et al. 2001, ApJ, 552, 473

Dennerl, K., Aschenbach, B., Briel, U. G., et al. 2004 [arXiv: astro-ph/0407637]

Fadda, D., Flores, H., Hasinger, G., et al. 2002, A\&A, 383, 838 Finoguenov, A., Briel, U. G., \& Henry, J. P. 2003, A\&A, 410, 777 Gilli, R., Cimatti, A., Daddi, E., et al. 2003, ApJ, 592, 721

Hasinger, G. 2003 [arXiv: astro-ph/0310804]

Hasinger, G., Burg, R., Giacconi, R., et al. 1998a, A\&A, 329, 482

Hasinger, G., Giacconi, R., Gunn, J. E., et al. 1998b, A\&A, 340, L27

Hasinger, G., Altieri, B., Arnaud, M., et al. 2001, A\&A, 365, L45

Kaastra, J. S., Lieu, R., Tamura, T., Paerels, F. B. S., \& den Herder, J. W. 2003, A\&A, 397, 445

Lehmann, I., Hasinger, G., Schmidt, M., et al. 2001, A\&A, 371, 833

Lumb, D. H., Warwick, R. S., Page, M., \& De Luca, A. 2002, A\&A, 389, 93

Mainieri, V., Bergeron, J., Hasinger, G., et al. 2002, A\&A, 393, 425

Mathur, S., Weinberg, D. H., \& Chen, X. 2003, ApJ, 582, 82

Mittaz, J., Lieu, R., Cen, R., \& Bonamente, M. 2004 [arXiv: astro-ph/0409661]

Nicastro, F. 2003, in IAU Symp.

Nicastro, F., Zezas, A., Drake, J., et al. 2002, ApJ, 573, 157

Read, A. M., \& Ponman, T. J. 2003, A\&A, 409, 395

Sołtan, A. M., Freyberg, M. J., \& Hasinger, G. 2002, A\&A, 395, 475

Strüder, L., Briel, U., Dennerl, K., et al. 2001, A\&A, 365, L18

Vikhlinin, A., McNamara, B. R., Forman, W., et al. 1998, ApJ, 502, 558

Wang, Q. D., Connolly, A., \& Brunner, R. 1997, ApJ, 487, L13+

Zappacosta, L., Mannucci, F., Maiolino, R., et al. 2002, A\&A, 394, 7

Zappacosta, L., Maiolino, R., Mannucci, F., Gilli, R., \& Schuecker, P. 2005, MNRAS, 357, 929 